\title{
SIGCHI Lifetime Research Award Talk-Seeing Past Looking Forward
}

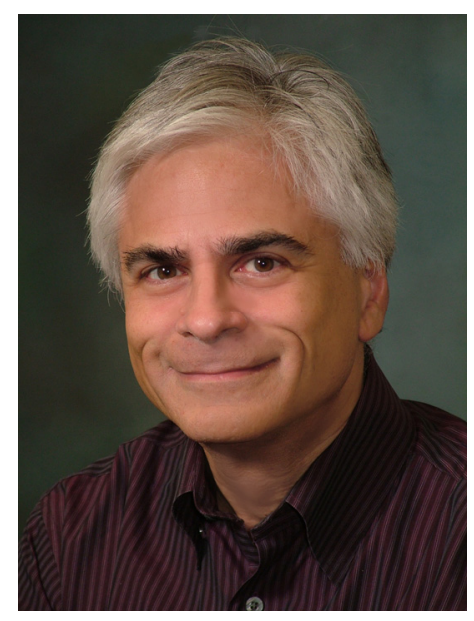

\section{Steven K. Feiner}

Department of Computer Science

Columbia University

New York, NY 10027, USA

feiner@cs.columbia.edu

http://www.cs.columbia.edu/ feiner
Permission to make digital or hard copies of part or all of this work for personal or classroom use is granted without fee provided that copies are not made or distributed for profit or commercial advantage and that copies bear this notice and the full citation on the first page. Copyrights copies bear this notice and the full citation on the first page. Copyrights
for third-party components of this work must be honored. For all other uses, contact the Owner/Author.

CHI'18 Extended Abstracts, April 21-26, 2018, Montréal, QC, Canada (C) 2018 Copyright is held by the owner/author(s).

ACM ISBN 978-1-4503-5621-3/18/04.

https://doi.org/10.1145/3170427.3185064

\begin{abstract}
I have long been interested in how computers can help people perform skilled tasks-a theme that underlies essentially all of my research. As the intentionally convoluted title of this talk implies, I will take a look back at some of this research to explain what came later, and to speculate on what might be next.

My earliest work as a graduate student was as part of a team developing novel hypermedia editing and presentation tools for technical documentation of equipment maintenance procedures. Understanding the effort involved in using these tools manually led to my dissertation, which explored rule-based techniques for the automated generation of 3D graphics that could ultimately replace the pictures found in maintenance manuals. Based on information about the task to be depicted, I designed a system that chose the objects to include, determined the level of detail at which to render them (e.g., adding more detail to disambiguate objects that could otherwise be confused with each other), highlighted important objects for emphasis, and created additional "metaobjects" (e.g., arrows to show actions). When I started as a faculty member, several of my students built on this research direction in both 3D and 2D domains, and we were soon collaborating with colleagues to develop generation approaches for
\end{abstract}


coordinated multimedia explanations that combined graphics with text.

At the same time, I was becoming excited by the potential of virtual reality (VR) to render objects stereoscopically and interact with them in 3D, in abstract domains as well as physical ones. And it was an easy jump from there to augmented reality (AR), cobbling together a simple monoscopic optical seethrough head-worn display. Our first AR system was a "hybrid" desktop window manager that embedded windows displayed on a flat panel display within a much larger virtual surround presented on the AR display. With that AR display now available as a tool, it became stunningly clear that so much of the effort we'd put into carefully crafting stand-alone pictures to explain tasks was unnecessary. If the user could instead look directly at the actual task domain, then a simple highlight or arrow, overlaid in situ, could show which button to push or knob to turn on a panel of controls.

Inspired by that observation, much of my lab's research has since addressed the design of AR user interfaces, for users indoors and outdoors, working alone and together. While AR does make some problems easier, it introduces difficult challenges! In classical user interfaces, designers and programmers control essentially all of what users experience; for example, determining the color at every pixel, accounting in advance for a limited set of variations. In contrast, AR celebrates and must work in perceptual context with the physical world. And that world is supremely fluid, in ways that are often unpredictable, as users and things in their environment move and change relative to each other. In response, the virtual user interface must change dynamically, as needed, to complement rather than interfere with the physical world; for example, a less important virtual object shouldn't obscure a more important object, whether physical or virtual.

Bearing this in mind and working not only in AR and $V R$, but in many kinds of desktop, mobile, and wearable user interfaces, my lab has tackled user interface design issues in domains such as healthcare, games, graphical editing, navigation, and equipment assembly. What comes next? I will conclude this talk by hazarding a few guesses (it is a dangerous sport!) about where some of this might lead.

\section{Author Keywords}

Human-computer interaction; Computer graphics; Knowledge-based graphics and multimedia; augmented reality; virtual reality; 3D user interfaces; wearable computing; health applications; computer games; information visualization.

\section{ACM Classification Keywords}

D.2.2 [Software Engineering]: Design Tools and Techniques-User interfaces; H.5.1 [Information Interfaces and Presentation]: Multimedia Information Systems-Artificial, augmented, and virtual realities; H.5.2 [Information Interfaces and Presentation]: User Interfaces-Input devices and strategies, Interaction styles; H.5.m [Information Interfaces and Presentation]: Miscellaneous; I.3.6 [Computer Graphics]: Methodology and Techniques-Interaction techniques; I.3.7 [Computer Graphics]: ThreeDimensional Graphics and Realism-Virtual reality; J.3 [Life and Medical Sciences]-Health, Medical information systems. 


\section{Bio}

Steven K. Feiner is a Professor of Computer Science at Columbia University, where he directs the Computer Graphics and User Interfaces Lab. His work has addressed a wide range of topics in human-computer interaction and computer graphics, including automated generation of graphics and multimedia, 3D and 2D user interfaces, wearable computing, health applications, computer games, and information visualization. His lab has been conducting virtual reality (VR), augmented reality (AR), and wearable computing research for over 25 years, designing and evaluating novel 3D interaction and visualization techniques, creating the first outdoor mobile AR system using a see-through head-worn display, and pioneering applications of AR to fields as diverse as tourism, maintenance, construction, and medicine.

Feiner is an IEEE Fellow, a member of the $\mathrm{CHI}$ Academy, and a recipient of the IEEE VGTC Virtual Reality Career Award and the IEEE ISMAR Career Impact Award. He and his students have won the ACM UIST Lasting Impact Award for their paper on supporting $2 \mathrm{D}$ windows in wearable $\mathrm{AR}$ and hypertextually linking them with each other and physical objects; the ISWC Early Innovator Award for their paper on outdoor mobile AR; and a number of best paper awards. Feiner has served as general chair or program chair for over a dozen ACM and IEEE conferences, including ACM UIST, has been a papers subcommittee chair for $A C M C H I$, and is a member of the editorial board of $A C M$ Transactions on ComputerHuman Interaction. He received an A.B. in Music and a Ph.D. in Computer Science, both from Brown
University, and is coauthor of two editions of Computer Graphics: Principles and Practice.

\section{Acknowledgments}

This work has been supported over the years, in large and small part and at different times, by the National Science Foundation, Office of Naval Research, Defense Advanced Research Projects Agency, National Library of Medicine, National Aeronautics and Space

Administration, Air Force Research Laboratory, New York State Center for Advanced Technology, and generous gifts and grants from Apple, Canon, Citicorp, Digital Image Design, Google, Hewlett-Packard, IBM, Institute for Information Industry, Intel, Meta, Microsoft Research, Mitsubishi, Nokia, NVIDIA, NYNEX, Raytheon, Verizon, VTT, and Vuzix. Any opinions, findings, and conclusions or recommendations expressed in this material are those of the author and do not necessarily reflect the views of any of the supporters listed above.

I thank my thesis advisor, Andy van Dam; my wife, Michele Page; my children, Maxwell and Alex; and my students and other collaborators, including Blaine Bell, Hrvoje Benko, Cliff Beshers, Gábor Blaskó, Andreas Butz, Norman Chin, Carmine Elvezio, Ajoy Fernandes, Hiroshi Furuya, Alon Grinshpoon, Sinem Güven, Steve Henderson, Tobias Höllerer, Christian Holz, Edward Ishak, Maryam Kamvar, David Kurlander, Jen-Shuo Liu , Gabrielle Loeb, Simon Lok, Blair MacIntyre, Kathy McKeown, Erick Méndez, Daniel Miau, Ohan Oda, Alex Olwal, John Pavlik, Shirin Sadri, Christian Sandor, Dorée Seligmann, Brian Smith, Pamela Smith, Mengu Sukan, Barbara Tversky, Tony Webster, Sean White, Lauren Wilcox, and Michelle Zhou. 